\title{
Month / Year of Diagnosis
}

National Cancer Institute

\section{Source}

National Cancer Institute. Month / Year of Diagnosis. NCI Thesaurus. Code C157434.

The month and the year of the diagnosis. 